\title{
A study of the pH dependence of electronically excited guanosine compounds by picosecond time-resolved infrared spectroscopy $\dagger$
}

\author{
David A. McGovern, ${ }^{a}$ Gerard W. Doorley, ${ }^{a}$ Aine M. Whelan, ${ }^{a}$ Anthony W. Parker, ${ }^{b}$ Michael Towrie, ${ }^{b}$ \\ John M. Kelly*a and Susan J. Quinn*a
}

\author{
Received 10th October 2008, Accepted 30th January 2009 \\ First published as an Advance Article on the web 6th March 2009 \\ DOI: 10.1039/b817756b
}

\begin{abstract}
The photophysical properties of $5^{\prime}$-guanosine monophosphate (5'-GMP) and polyguanylic acid $\{$ poly $(\mathrm{G})\}$ in $\mathrm{D}_{2} \mathrm{O}$ solutions of varying $\mathrm{pH}$ have been studied using picosecond transient infrared absorption spectroscopy. Whereas in neutral or weakly alkaline solution only the vibrationally excited electronic ground state of $5^{\prime}$-GMP is observed, in acidic solution the relatively long-lived ( $\left.229 \pm 20 \mathrm{ps}\right)$ electronic excited state of protonated 5'-GMP, which possesses strong absorptions at 1517 and $1634 \mathrm{~cm}^{-1}$, could be detected. The picosecond transient behaviour of polyguanylic acid in acidic solution is also very different from that of the polynucleotide in neutral solution due not only to the protonation of guanine moieties yielding the protonated excited state but because of the disruption of the guanine stacks which are present in the species in neutral solution.
\end{abstract}

\section{Introduction}

Picosecond time-resolved infrared (ps-TRIR) $)^{1-8}$ is emerging as a powerful technique for the study of the photophysical processes in nucleic acids. TRIR has followed on from the study of DNA systems by both UV-vis transient absorption and fluorescence techniques. ${ }^{10-13}$ Our work seeks to understand how photochemically driven reactivity is altered by environmental perturbations and/or interaction with other nucleic acid bases, as found naturally in polymeric and stacked forms of the bases. To this end guanine nucleotides and polynucleotides are particularly interesting. Guanine has the lowest ionization potential of the four DNA bases and consequently is most susceptible to photooxidation, a process that leads to the formation of radical cations which may lead to DNA damage, mutation and the onset of cancer.,14 Guanine is also interesting for its ability, through Hoogsteen binding, to form tetrad structures (1).

The mononucleotides 5'-GMP and 5'-dGMP are only very weakly luminescent and this has been attributed to the ultra-short lifetime (860 fs for $5^{\prime}$-dGMP from fluorescence upconversion $)^{15}$ of the emitting singlet excited state, caused by very rapid internal conversion to form a vibrationally excited electronic ground state. ${ }^{16}$ The relaxation of this latter species through coupling to the solvent occurs on the picosecond time scale and has previously been characterised by ps-TRIR measurements. ${ }^{1,3}$ However, it is known that very simple modifications of nucleic acids, such as chemical substitution or the presence of a ribose group or protonation, may significantly change the excited state lifetimes. ${ }^{17}$ In this light the protonation of $5^{\prime}$-GMP to form $5^{\prime}-\mathrm{GMPH}^{+}$(2)

${ }^{a}$ School of Chemistry and Centre for Chemical Synthesis and Chemical Biology, Trinity College,Dublin 2, Ireland.E-mail: jmkelly@tcd.ie, quinnsu@tcd.ie; Fax: +35 316712826; Tel: +35 318961947

${ }^{b}$ Central Laser Facility, Science \& Technology Facilities Council, Rutherford Appleton Laboratory, Harwell Science and Innovation Campus, Didcot, Oxfordshire, UK OX11 $0 Q X$

$\dagger$ Electronic supplementary information (ESI) available. See DOI: $10.1039 / \mathrm{b} 817756 \mathrm{~b}$ $\left(\mathrm{p} K_{\mathrm{a}}=2.3\right)$ is known to have a dramatic effect on the lifetime of the electronic excited state. The protonated form is significantly more fluorescent than the neutral base. In the first transient spectroscopy study of this system, Fujiwara et al. ${ }^{18}$ used a synchroscan streak camera to monitor the effect of $\mathrm{pH}$ on the lifetimes of the excited states of guanine, guanosine and 5'-GMP. They observed that the lifetime of the excited state of $5^{\prime}$-GMP was significantly longer at acidic $\mathrm{pH}$ ( $c a .200 \mathrm{ps}$ ) than for the neutral molecule. Guanosine (Guo) at pH 3 has also been investigated by Peon and Zewail using femtosecond up-conversion transient fluorescence methods. ${ }^{15}$ Here, three lifetime components were recorded $\left[\tau_{1}=\right.$ $660 \mathrm{fs}(67 \%), \tau_{2}=3.4 \mathrm{ps}(18 \%)$ and $\left.\tau_{3}=209 \mathrm{ps}(15 \%)\right]$. The longlived component was in agreement with fluorescence observations made using a streak camera and attributed to the $\mathrm{GuoH}^{+}$species. The subpicosecond component was the same as that recorded for the unprotonated Guo in neutral solution and as such attributed to the presence of this species. The 3.4 ps component was attributed to relaxation processes within the $S_{1}$ state of protonated guanine. Pecourt et al. in their femtosecond pump-probe transient absorption study of $5^{\prime}-\mathrm{GMPH}^{+}$again observed the presence of the long-lived component, $\tau=194 \mathrm{ps}^{16}$ These results are therefore all consistent with the lifetime of the fluorescing singlet excited state of $5^{\prime}-\mathrm{GMPH}^{+}$being $c a$. $200 \pm 20$ ps.
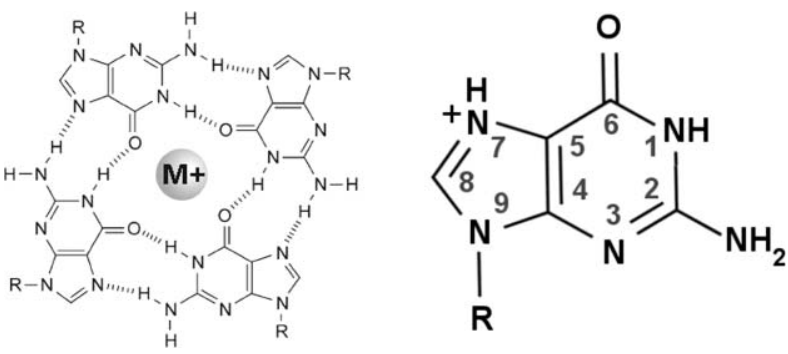

Under certain conditions, guanine-based Hoogsteen tetrads may form extended structures through stacking interactions. ${ }^{19,20}$ Such stacking interactions have been considered in light of the presence of repeat runs of guanine in the human telomeric 
sequence found at the end of chromosomal DNA. ${ }^{21}$ FTIR is a powerful technique for probing the DNA secondary structures present in solution, as bands in the region $1500-1700 \mathrm{~cm}^{-1}$ are very sensitive to stacking interactions. For example, the characteristic IR bands of 5'-GMP undergo marked changes in frequency and intensity when guanine is present in ordered structures. ${ }^{20}$ In our initial ps-TRIR study of guanine-rich systems we observed that systems containing stacked guanine tetrads, such as $5^{\prime}$-GMP at high concentration or polyguanylic acid [poly $(\mathrm{G})]$ showed a longlived transient in addition to the vibrationally excited ground state. This transient species was assigned to an excimer within the stacked structure. ${ }^{3}$

In the present publication, we use ps-TRIR to follow the excited state processes in 5'-GMP in neutral, acidic and alkaline $\mathrm{D}_{2} \mathrm{O}$ solutions. We then consider the effect protonation of the guanine base has on the photodynamics of poly $(\mathrm{G})$, a system that is known to adopt interesting secondary structures based on stacked guanine tetrads. ${ }^{3}$ Note that under our experimental conditions all exchangeable protons are replaced by deuterons.

\section{Experimental}

\section{Materials and sample handling}

The $5^{\prime}-\mathrm{H}_{2} \mathrm{GMP}$ was supplied by MP Biomedicals Inc. as the $5^{\prime}-\mathrm{H}_{2}$ GMP free acid, purity $98-100 \%$. The $5^{\prime}-\mathrm{Na}_{2} \mathrm{GMP}$, polyguanylic, phosphate buffer components, $\mathrm{D}_{2} \mathrm{O}$ and other reagents were all supplied by Sigma-Aldrich. Samples were prepared by weighing out an amount of the nucleotide base or polynucleotide into an eppendorf followed by the addition of a known amount of $\mathrm{D}_{2} \mathrm{O}$ or $\mathrm{D}_{2} \mathrm{O}$ buffer solution and the concentration was determined by UV measurements.

\section{Picosecond time-resolved infrared (ps-TRIR) experiments}

The ps-TRIR experiments were carried out at the Central Laser Facility of the Rutherford Appleton Laboratory. This apparatus has been described previously ${ }^{9}$ and a brief description is given in the ESI. $\uparrow$ The sample was excited with a $150 \mathrm{fs} 267 \mathrm{~nm}$ pulse of energy approximately $2 \mu \mathrm{J}$ with a spot size of between $150-200 \mu \mathrm{m}$. The data were collected in a number of $150 \mathrm{~cm}^{-1}$ wide spectral windows centred at approximately 1625 and $1565 \mathrm{~cm}^{-1}$ using the delay line for optical delays between 2 ps and $1.5 \mathrm{~ns}$, normally at $2,3,4,5,6.5,8,10,12.5,15,20,35,50,100,150,200,500,1000$ and $1500 \mathrm{ps}$. The sample in $\mathrm{D}_{2} \mathrm{O}$ was placed between two $25 \mathrm{~mm}$ $\mathrm{CaF}_{2}$ plates in a Harrick cell. For low concentration experiments a $12 \mu \mathrm{m}$ Teflon spacer was used and no spacer was used in the case of high concentration experiments. Ground state UV-vis and FTIR spectra were recorded before and after all experiments using a Perkin Elmer Lambda 2 and a Nicolet Avatar 360 respectively to ensure that no photodegradation occurred during the ps-TRIR experiments.

\section{Results \\ 5'-GMP and 5'-GMPD ${ }^{+}$}

$5^{\prime}$-GMP excited by a $150 \mathrm{fs}$ pulse of $267 \mathrm{~nm}$ radiation shows results very similar to those of $5^{\prime}$-dGMP which has previously been characterised at $\mathrm{pH} 7$ by ps-TRIR ${ }^{1,3}$ (see also ESI). In these experiments the bleached bands of the ring $\left(1581 \mathrm{~cm}^{-1}\right)$ and carbonyl $\left(1669 \mathrm{~cm}^{-1}\right)$ stretches were found to decay rapidly $(<6 \mathrm{ps})$. This rapid decay was attributed to cooling of the vibrationally hot ground state after relaxation from the electronically excited state. Interestingly, the rate of recovery to the thermally equilibrated ground state is different for the ring-based vibration (lifetime $3.1 \pm 0.3 \mathrm{ps})$ than for the carbonyl-based vibration $(4.7 \pm 0.4 \mathrm{ps})$, suggesting that there is some degree of mode-specific interaction with the solvent. ${ }^{3}$ The IR transient absorptions also show a characteristic tracking behaviour with the absorption maximum moving to higher wavenumber as the transient decays. ${ }^{3}$

To explore the influence of $\mathrm{pH}$ on this system, $5^{\prime}$-GMP was first monitored in acidic $\mathrm{D}_{2} \mathrm{O}$ solution (pD 2) at various delays after $267 \mathrm{~nm}$ excitation (Fig. 1). The behaviour observed at this $\mathrm{pH}$ was found to be very different from that in neutral solution. The protonation of $5^{\prime}-\mathrm{GMP}$, at the $\mathrm{N} 7$ position of the imidazole ring to form $5^{\prime}-\mathrm{GMPD}^{+}$, results in major changes in the ground state FTIR spectrum. The ring band at $1578 \mathrm{~cm}^{-1}$ is accompanied by an additional band at $1608 \mathrm{~cm}^{-1}$ and the carbonyl band is found to shift from 1669 to $1689 \mathrm{~cm}^{-1}$. The three bands manifest themselves as bleaches in the ps-TRIR and two intense transient absorption bands are present at 1517 and $1634 \mathrm{~cm}^{-1}$. Both transient bands for this excited state occur at lower frequency than for the ground state. While assignment of the transient bands will require examination by computational methods, we provisionally assign these to the purine-ring based $\left(1517 \mathrm{~cm}^{-1}\right)$ and carbonyl based $\left(1634 \mathrm{~cm}^{-1}\right)$ vibrations as in the ground state. In the case of the carbonyl this suggests an excited state with less double bond character than the ground state.

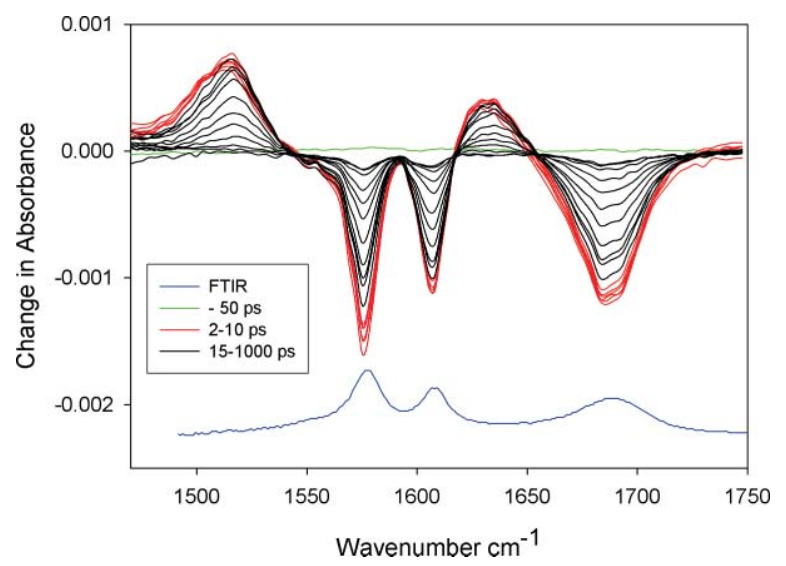

Fig. 1 ps-TRIR of $10 \mathrm{mM} 5^{\prime}-\mathrm{Na}_{2} \mathrm{GMP}$ under acidic conditions, $0.132 \mathrm{M}$ $\mathrm{H}_{3} \mathrm{PO}_{4}$ in $\mathrm{D}_{2} \mathrm{O}$, with FTIR below (blue). Spectra at delays of $-25 \mathrm{ps}$ (green), 2-10 ps (red) and 15-1000 ps (black).

Analysis of the kinetics of the bleach and transient decays reveals a monoexponential recovery to the ground state, with a lifetime of $229 \pm 20$ ps (Fig. 2). This is considerably longer than observed for $5^{\prime}$-GMP under neutral conditions and is consistent with the presence of a protonated excited state, which has previously been identified by fluorescence ${ }^{15,18}$ and transient visible absorption spectroscopy. ${ }^{17}$

Next $5^{\prime}-\mathrm{H}_{2} \mathrm{GMP}$ was studied in unbuffered $\mathrm{D}_{2} \mathrm{O}$ solution. Under these conditions a mixture of the protonated and neutral species is present, as can be seen from the FTIR (bottom of Fig. 3a). The ps-TRIR indicates the presence of two different decaying 


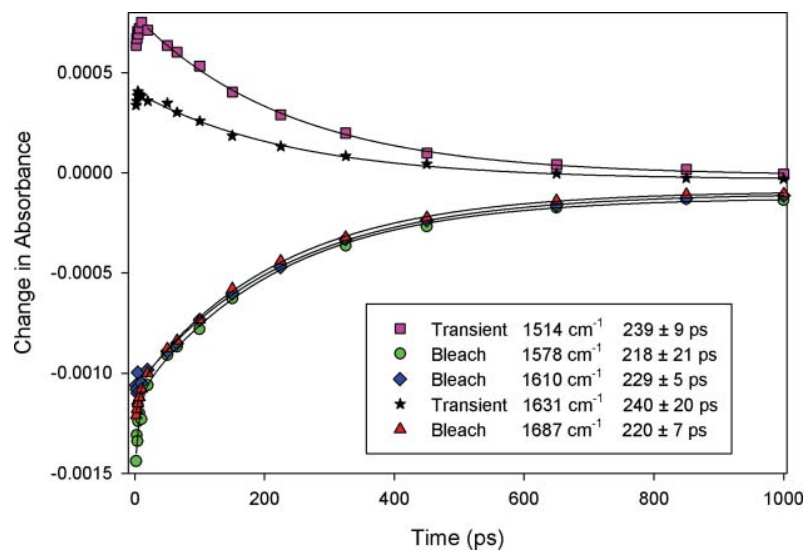

Fig. 2 Kinetic analysis of ps-TRIR data for $10 \mathrm{mM} 5^{\prime}-\mathrm{Na}_{2} \mathrm{GMP}$ under acidic conditions, $0.132 \mathrm{M} \mathrm{H}_{3} \mathrm{PO}_{4}$ in $\mathrm{D}_{2} \mathrm{O}$. Kinetics recorded at the carbonyl and ring bleach and associated transient positions $1687 \mathrm{~cm}^{-1}(\mathbf{\Delta})$, $1631 \mathrm{~cm}^{-1}(\star), 1610 \mathrm{~cm}^{-1}(\diamond), 1578 \mathrm{~cm}^{-1}(\bullet)$ and $1514 \mathrm{~cm}^{-1}(\mathbf{\square}) .1578$ and $1687 \mathrm{~cm}^{-1}$ traces calculated over the range of $2-1000$ ps with 1514,1610 , and $1631 \mathrm{~cm}^{-1}$ calculated over the range $20-1000 \mathrm{ps}$.
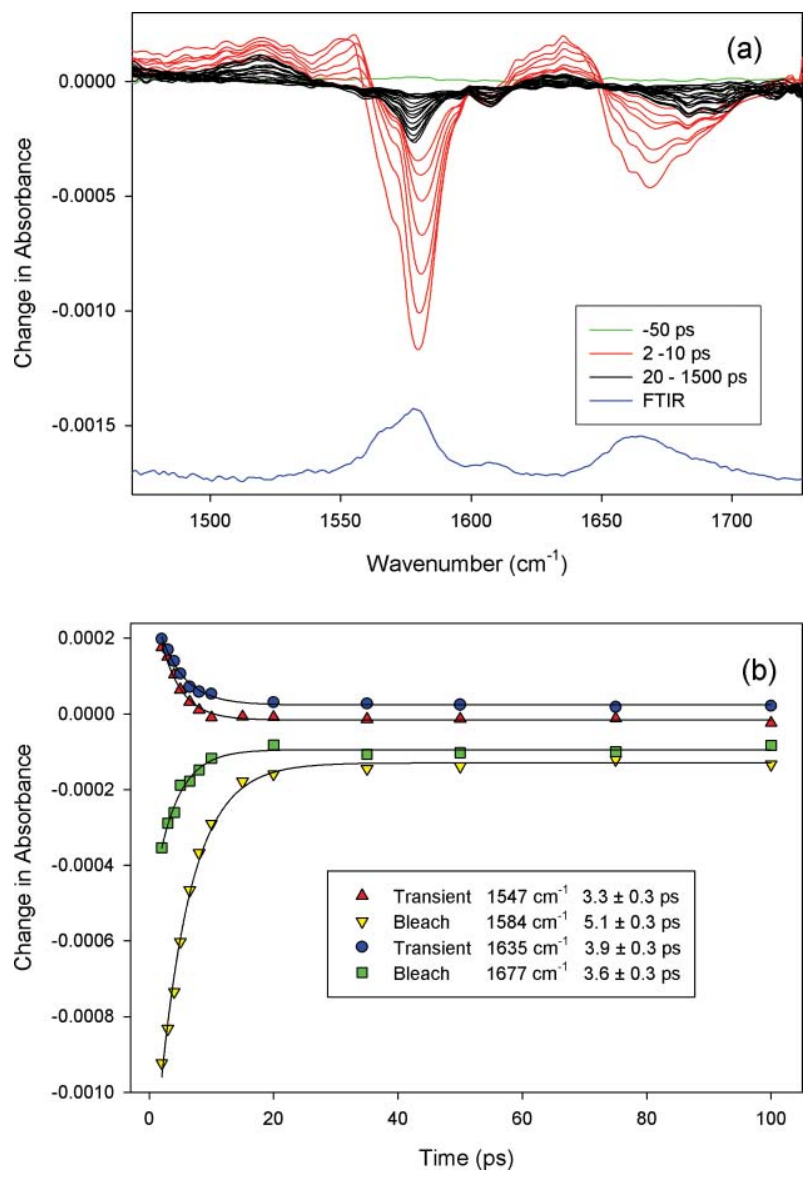

Fig. 3 ps-TRIR and FTIR of $10 \mathrm{mM} 5^{\prime}-\mathrm{H}_{2}$ GMP in unbuffered $\mathrm{D}_{2} \mathrm{O}$. (a) Spectra at delays of $-25 \mathrm{ps}$ (green), 2-10 ps (red) and 20-1500 ps (black). (b) Kinetics recorded at the carbonyl and ring bleach and associated transient positions: $1677 \mathrm{~cm}^{-1}(\boldsymbol{\square}), 1635 \mathrm{~cm}^{-1}(\boldsymbol{\bullet}), 1584 \mathrm{~cm}^{-1}(\boldsymbol{\nabla})$ and $1547 \mathrm{~cm}^{-1}(\mathbf{\Delta})$.

species, one fast (1580 and $1669 \mathrm{~cm}^{-1}$, appears in red) and one slow (1578, 1608 and $c a .1689 \mathrm{~cm}^{-1}$, appears in black). This difference in behaviour is most striking in the carbonyl region $\left(1650-1700 \mathrm{~cm}^{-1}\right)$ of the ps-TRIR spectra (Fig. 3a), where the vibrationally excited ground state (red component of the transient) is easily discernible from the longer-lived protonated excited state (black component of the transient). Analysis of the bands characteristic of the neutral species yields short decay kinetics. For example for the carbonyl $\left(1677 \mathrm{~cm}^{-1}\right)$ and ring $\left(1584 \mathrm{~cm}^{-1}\right)$ bleached bands, lifetimes of $3.6 \pm 0.3$ ps and $5.1 \pm 0.5$ ps were found. The decay times of the corresponding transient absorptions also display similar kinetics with decay times of $3.5 \pm 0.3$ ps and $3.3 \pm 0.3$ ps observed at $1635 \mathrm{~cm}^{-1}$ and $1547 \mathrm{~cm}^{-1}$ respectively. In turn, examination of the features associated with protonated species give long-lived lifetimes in agreement with those reported above. For example, the carbonyl bleach at $1690 \mathrm{~cm}^{-1}$ recovers with a lifetime of $224 \pm 23 \mathrm{ps}$ (measured between 20-1500 ps) while the associated transient at $1635 \mathrm{~cm}^{-1}$ decays with a lifetime of $205 \pm 41 \mathrm{ps}$. The transient feature at $1517 \mathrm{~cm}^{-1}$ displays features of the neutral and protonated form with a short decay times of $3.1 \pm 0.5(56 \%)$ and a long $243 \pm$ $24(44 \%)$ respectively. The results of the kinetic analysis at each of the transient absorption and bleaching maxima are presented in Table 1.

5'-GMP was also studied under mildly alkaline conditions ( $\mathrm{pH}$ 8.5). The results obtained at this $\mathrm{pH}$ were found to be very

Table 1 Decay times for $5^{\prime}$-GMP in neutral solution and poly(G) in neutral and acidic solution

\begin{tabular}{|c|c|c|}
\hline Guanine system & $\begin{array}{l}\text { FTIR/ } \\
\mathrm{cm}^{-1}\end{array}$ & Lifetime (ps) and $\%$ contribution \\
\hline \multirow[t]{2}{*}{$5^{\prime}$-GMP neutral ${ }^{a}$} & 1577 & 1553 (T) $3.5 \pm 0.3, \mathbf{1 5 8 7}$ (B) $4.7 \pm 0.4$ \\
\hline & 1662 & 1642 (T) $3.3 \pm 0.3,1676$ (B) $3.1 \pm 0.3$ \\
\hline \multirow{5}{*}{$\begin{array}{l}5^{\prime}-\mathrm{Na}_{2} \text { GMP in } \\
0.132 \mathrm{M} \mathrm{H}_{3} \mathrm{PO}_{4}{ }^{b}\end{array}$} & 1577 & 1514 (T) $239 \pm 9$ \\
\hline & 1607 & 1610 (B) $229 \pm 5$ \\
\hline & 1688 & 1578 (В) $6.5 \pm 2.2(27 \%), 218 \pm 21(73 \%)$ \\
\hline & & 1631 (T) $240 \pm 20$ \\
\hline & & 1687 (B) $7.0 \pm 1.5(15 \%), 220 \pm 7(85 \%)$ \\
\hline \multirow{7}{*}{$5^{\prime}-\mathrm{H}_{2} \mathrm{GMP}$ in $\mathrm{D}_{2} \mathrm{O}^{c}$} & 1577 & 1517 (T) $3.1 \pm 0.5(56 \%), 243 \pm 24(44 \%)$ \\
\hline & 1607 & 1547 (T) $3.3 \pm 0.3$ \\
\hline & 1662 & 1579 (В) $3.3 \pm 0.1(89 \%), 220 \pm 27(11 \%)$ \\
\hline & 1688 & 1584 (B) $5.1 \pm 0.3,194 \pm 24$ \\
\hline & & 1635 (T) $3.5 \pm 0.3(83 \%), 205 \pm 41(17 \%)$ \\
\hline & & 1677 (B) $3.6 \pm 0.3$ \\
\hline & & 1690 (B) $224 \pm 23$ \\
\hline \multirow[t]{5}{*}{$\operatorname{Poly}(\mathrm{G})^{d}$} & 1581 & 1553 (T) $3.6 \pm 0.7(68 \%), 35 \pm 9(28 \%)$ \\
\hline & 1610 & 1599 (T) $3.7 \pm 0.5(59 \%), 36 \pm 4(41 \%)$ \\
\hline & 1687 & 1608 (B) $2.5 \pm 1.4(60 \%), 52 \pm 18(40 \%)$ \\
\hline & & 1648 (T) $4.5 \pm 0.6(72 \%), 36 \pm 8(28 \%)$ \\
\hline & & 1687 (B) $5.1 \pm 1.4(47 \%), 37 \pm 6(53 \%)$ \\
\hline \multirow[t]{7}{*}{$\operatorname{Poly}(\mathrm{G}) \mathrm{H}^{+e}$} & 1577 & 1559 (T) $9 \pm 2(58 \%), 133 \pm 30(42 \%)$ \\
\hline & 1603 & 1584 (В) $24 \pm 9(56 \%), 190 \pm 75(44 \%)$ \\
\hline & 1673 & 1606 (B) $18 \pm 4(70 \%), 201 \pm 85(30 \%)$ \\
\hline & 1698 & 1640 (T) $17 \pm 6(46 \%), 92 \pm 25(54 \%)$ \\
\hline & & 1672 (B) $18 \pm 4(80 \%), 190 \pm 131(20 \%)$ \\
\hline & & 1689 (B) $16 \pm 4(69 \%), 118 \pm 48(31 \%)$ \\
\hline & & 1702 (B) $13 \pm 4(57 \%), 104 \pm 31(43 \%)$ \\
\hline
\end{tabular}

$(\mathrm{B})=$ bleach; $(\mathrm{T})=$ transient.${ }^{a}$ Range of $2-100$ ps. ${ }^{b}$ Range of $20-1000 \mathrm{ps}$ for long single exponential times and for biexponential result range of $2-1000$ ps. ${ }^{c}$ Short single exponential range of $2-100 \mathrm{ps}$, long single exponential range of $20-1500 \mathrm{ps}$ and any biexponential range of 2-1500 ps. ${ }^{d}$ Range of $2-500 \mathrm{ps}^{e}{ }^{e}$ The range was $3-500$ for $1640 \mathrm{~cm}^{-1}$ and $3-1500 \mathrm{ps}$ for all other bands. 
similar to those observed at neutral pH (ESI, Fig. S1a $\uparrow$ ). Regions of transient absorption and depletion ('bleaching') of the ground state ring and carbonyl bands are present. The bleached bands were found to recover with lifetimes of $\tau=2.7 \pm 0.3 \mathrm{ps}\left(1675 \mathrm{~cm}^{-1}\right)$ and $5.2 \pm 0.5 \mathrm{ps}\left(1587 \mathrm{~cm}^{-1}\right)$ respectively (ESI, Fig. S1b $\left.\dagger\right)$. These data are comparable to the results found for $10 \mathrm{mM} 5^{\prime}$-GMP in neutral conditions \{i.e. $3.1 \pm 0.3 \mathrm{ps}\left(1676 \mathrm{~cm}^{-1}\right)$ and $4.7 \pm$ $\left.0.5 \mathrm{ps}\left(1587 \mathrm{~cm}^{-1}\right)\right\}{ }^{1,3}$ The results complement those already in the literature and clearly show that chemical changes can be readily identified and monitored using TRIR. However, as biological systems are based on polynucleotide sequences we now consider the effect of protonation on a guanine-based polymer as seen by ps-TRIR.

\section{$\operatorname{Poly}(G)$ and $\operatorname{poly}\left(\mathrm{GH}^{+}\right)$}

Guanine bases are known to self-assemble in solution to form stacked tetrads through Hoogsteen hydrogen bonding. ${ }^{19}$ This phenomenon is also found for $\operatorname{poly}(\mathrm{G})$ which forms a fourstranded helical structure. The formation of this structure is readily observable using circular dichroism. ${ }^{21}$ The spectrum is dominated by an exciton couplet characterized by a positive band at $c a$. $260 \mathrm{~nm}$ with a shoulder at $c a .290 \mathrm{~nm}$, and a negative maximum at $c a .240 \mathrm{~nm}$ (Fig. 4). The presence of these bands under the experimental conditions indicates the existence of the assembled form in solution.

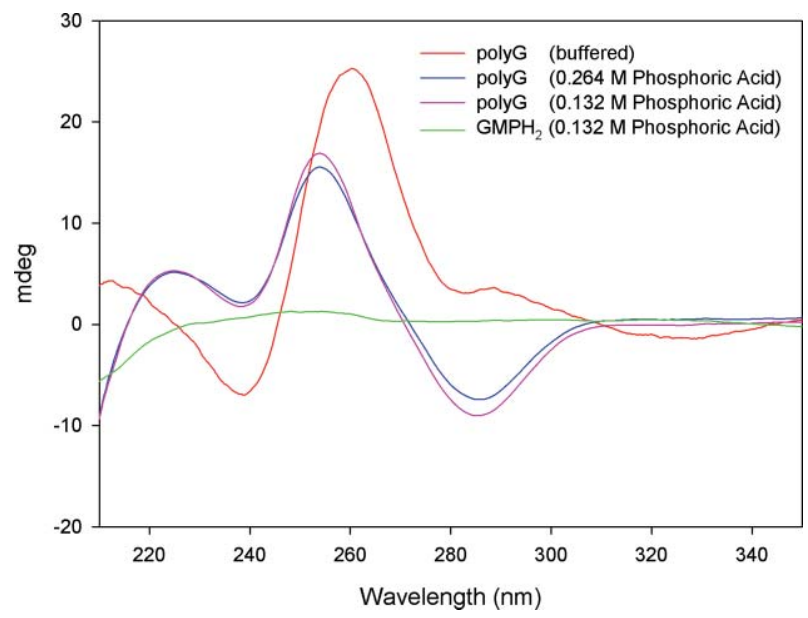

Fig. 4 Circular dichroism of $10 \mathrm{mM} 5^{\prime}-\mathrm{H}_{2} \mathrm{GMP}$ in $0.132 \mathrm{M} \mathrm{H}_{3} \mathrm{PO}_{4}$, $11.3 \mathrm{mM}$ poly $(\mathrm{G})$ in $0.132 \mathrm{M} \mathrm{H}_{3} \mathrm{PO}_{4}, 11.3 \mathrm{mM}$ poly $(\mathrm{G})$ in $0.264 \mathrm{M} \mathrm{H}_{3} \mathrm{PO}_{4}$, $11.3 \mathrm{mM}$ poly $(\mathrm{G})$ in $50 \mathrm{mM}$ potassium phosphate buffer, all samples in $\mathrm{D}_{2} \mathrm{O}$.

The presence of guanine stacked tetrads is also readily identified by FTIR spectra. The guanine carbonyl and ring stretches both undergo changes upon the formation of the stacked Hoogsteen hydrogen bonded guanines..$^{20} \mathrm{~A}$ narrowing of the carbonyl band is observed and the band shifts to higher wavenumbers $\left(1663 \mathrm{~cm}^{-1}\right.$ to $1688 \mathrm{~cm}^{-1}$ ), while the ring bands are found to undergo significant hypochroism (see ESI, Fig. S2†). In Fig. 5a and 5c the ps-TRIR, 2 ps after excitation, of poly $(\mathrm{G})$ and $5^{\prime}-\mathrm{GMP}$, at neutral $\mathrm{pH}$ are compared. As expected from the FTIR, the bleaching of the ring-based vibrations of poly $(\mathrm{G})$ are noticeably suppressed and there is a sharpening of the carbonyl vibration, which shifts from $1669 \mathrm{~cm}^{-1}$ to $1690 \mathrm{~cm}^{-1}$, indicating the formation and stacking of

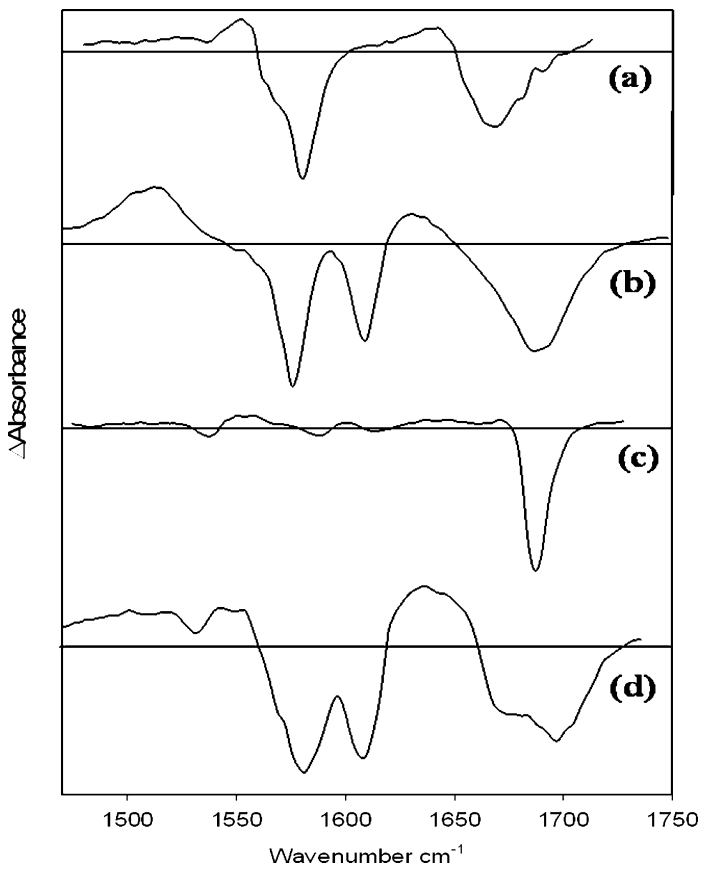

Fig. 5 ps-TRIR spectra all recorded at 2 ps of (a) $10 \mathrm{mM} \mathrm{5'-GMP} \mathrm{in}$ $50 \mathrm{mM}$ potassium phosphate $\mathrm{D}_{2} \mathrm{O}$ buffer at $\mathrm{pH}$ 7, (b) $10 \mathrm{mM}$ free acid 5'-GMP in $0.132 \mathrm{M} \mathrm{H}_{3} \mathrm{PO}_{4}$ in $\mathrm{D}_{2} \mathrm{O}$, (c) $11.4 \mathrm{mM}$ poly(G) in $50 \mathrm{mM}$ potassium phosphate $\mathrm{D}_{2} \mathrm{O}$ buffer at $\mathrm{pH} 7$ and (d) $11.3 \mathrm{mM}$ poly(dG) in $0.132 \mathrm{M} \mathrm{H}_{3} \mathrm{PO}_{4}$ in $\mathrm{D}_{2} \mathrm{O}$.

guanine tetrads. ${ }^{20}$ The kinetic behaviour of the poly $(\mathrm{G})$ also differs markedly from that of $5^{\prime}$-GMP in dilute solution. (However, it is similar to that of the stacked system formed in the presence of high $5^{\prime}$-GMP concentration. ${ }^{3}$ ) Analysis of the recovery of the carbonyl bleach at $1687 \mathrm{~cm}^{-1}$ reveals a biexponential process with a short component of $5 \pm 1 \mathrm{ps}(47 \%)$ and a longer component of $37 \pm$ $6 \mathrm{ps}(53 \%)$. Interestingly, whilst the short lifetime is comparable to that observed for the vibrationally hot ground state of $5^{\prime}$-GMP there is an absence of any tracking of the bands. This phenomenon has been recently observed in other polymer systems and may be explained by a delocalization of the excess vibrational energy over neighbouring bases in the polymer form. ${ }^{8}$ The longer-lived species is assigned to an excimeric excited state. ${ }^{3}$

Next the effect of protonation of the poly $(\mathrm{G})$ has been studied in $\mathrm{D}_{2} \mathrm{O}$. The CD of poly $(\mathrm{G})$ (Fig. 4) shows that in the presence of acid there is a change in sign of the band at $290 \mathrm{~nm}$ and a shift in the intensity and position of the positive band at $260 \mathrm{~nm}$ to $255 \mathrm{~nm}$. Furthermore, the band at $240 \mathrm{~nm}$ disappears. These results are in good agreement with those observed previously under similar conditions. ${ }^{21}$ The spectra are closely similar at both $0.132 \mathrm{M}$ and $0.264 \mathrm{M} \mathrm{H}_{3} \mathrm{PO}_{4}$, showing that the extent of protonation is independent of the acid concentration in this range. As expected, the decrease in $\mathrm{pH}$ results in changes in the ground state FTIR spectrum. The ring vibrations are no longer suppressed with two bands observed at 1577 and $1603 \mathrm{~cm}^{-1}$ and the region associated with the carbonyl bond is dominated by a broad structured band at $1700 \mathrm{~cm}^{-1}$ with a shoulder of lower intensity between $1663-75 \mathrm{~cm}^{-1}$ (Fig. 6). These changes are attributed to protonation of guanine within the polymer and a disruption of the tetrad stacking. The ps-TRIR, 2 ps after excitation, of poly(G) under acidic conditions is shown in Fig. 5d. All four ground state IR absorption bands 


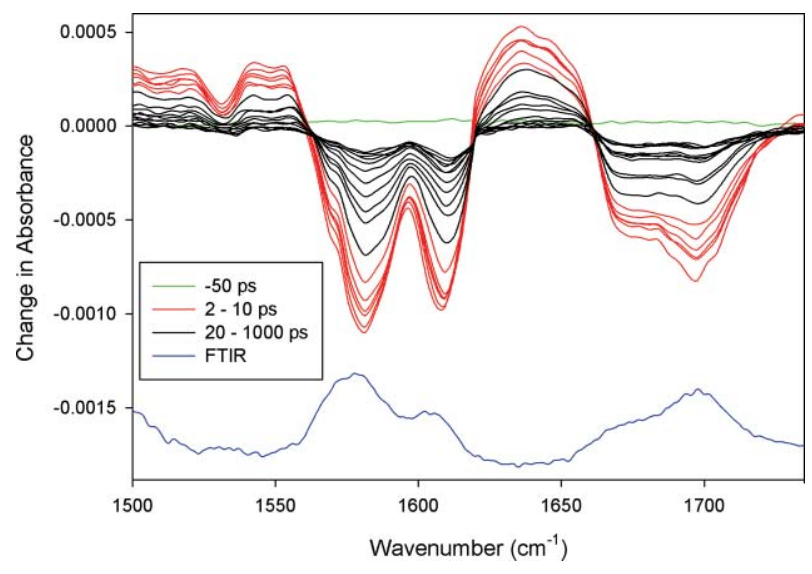

Fig. 6 (a) ps-TRIR of $11.3 \mathrm{mM}$ poly $(\mathrm{G})$ under acidic conditions, $0.132 \mathrm{M}$ $\mathrm{H}_{3} \mathrm{PO}_{4}$ in $\mathrm{D}_{2} \mathrm{O}$ with FTIR below. Spectra at delays of -50 ps (green), $2-10 \mathrm{ps}$ (red) and 20-1000 ps (black). Spectra recorded in windows centred at $1557 \mathrm{~cm}^{-1}$ and $1633 \mathrm{~cm}^{-1}$ and joined at $1561 \mathrm{~cm}^{-1}$.

appear as bleaches in the ps-TRIR spectra and, in addition, there are two strongly absorbing transient signals observed, one between $1500-1550 \mathrm{~cm}^{-1}$ and a second broad band centred at $1640 \mathrm{~cm}^{-1}$. These bands are assigned to a ring-based vibration and a carbonyl vibration in the excited state respectively. Significantly, both the bleaching and transient absorption regions of the spectrum are found to be similar to that observed for $5^{\prime}-\mathrm{GMPD}^{+}$at the same time of 2 ps (Fig. 5b).

The ps-TRIR profiles of protonated poly $(\mathrm{G})$ recorded at delays 2-1500 ps are shown in Fig. 6. Examination of the data reveals complex kinetics, with biexponential decay and recovery, observed in the bleach and transient features (Table 1). The faster decaying component is markedly longer-lived and the slower-decaying species is significantly shorter-lived, than their counterparts in neutral poly $(G)$. The short times are possibly extended due to the superposition of a number of short-lived states which result from incomplete protonation.

\section{Discussion}

The mechanism by which nucleic acids dissipate excess energy is a subject of increased interest. ${ }^{12,23}$ In the case of $5^{\prime}$-GMP it is now recognised that the initially formed ${ }^{1} \pi \pi^{*}$ state undergoes rapid internal conversion at a conical intersection forming the electronic ground state with considerable excess vibrational energy. ${ }^{16}$ It is this 'hot' ground-state transient behaviour that can be monitored at early, $<5$ ps, times in our ps-TRIR experiments. ${ }^{1,3}$ The speed of energy loss from this species has been shown to be dependent on the vibrational mode involved and is slightly faster for the vibration associated with the carbonyl group than for that of the ring $(\mathrm{C}=\mathrm{C}, \mathrm{C}=\mathrm{N}){ }^{3}$ The current study further supports the contention that for $5^{\prime}$-GMP the species observed under these neutral/weakly alkaline conditions is the vibrationally excited ground electronic ground state.

By contrast in strongly acidic $\mathrm{D}_{2} \mathrm{O}$ solution, quite different behaviour is observed. Under these conditions the ground state is protonated at the 7-position, giving $5^{\prime}-\mathrm{GMPD}^{+} .{ }^{24}$ This results in removal of electronic charge from the pyrimidine ring. The subsequent decoupling of the $\mathrm{C}=\mathrm{O}$ mode with the solvent through less hydrogen bonding leads to a shifting of the $\mathrm{C}=\mathrm{O}$ mode by $21 \mathrm{~cm}^{-1}$, representative of more double bond character. The transient observed following $267 \mathrm{~nm}$ excitation shows that the excited state deactivates with a lifetime of $229 \pm 25$ ps. This is much longer than that observed from 5'-GMP in neutral solution and is closely similar to that observed by fluorescence ${ }^{15}$ and transient visible absorption. ${ }^{16,17}$ This protonated excited state is characterised by two strong absorption bands in the IR at 1518 and $1634 \mathrm{~cm}^{-1}$. At this stage, in the absence of computational studies we are unable to unambiguously assign these features that probably relate to the excited state being a ${ }^{1} \pi \pi^{*}$ state of $5^{\prime}-\mathrm{GMPD}^{+}$. Interestingly, there is little evidence of the band tracking that is characteristic of vibrational cooling., 2,36,25 This would suggest that either when the protonated excited state is formed, it does so with only a small amount of excess vibrational energy or that the solvent coupling is sufficiently increased in this species that vibrational relaxation is extremely efficient, and as a result we are unable to observe the tracking behaviour within the time-resolution of our experiments.

In unbuffered solutions of $5^{\prime}-\mathrm{H}_{2}$ GMP both the $5^{\prime}$-GMP and $5^{\prime}-\mathrm{GMPD}^{+}$are present. Probing the transient absorption of this mixture yields spectra and kinetics that are entirely consistent with the behaviour expected for both species. The characteristic shortlived vibrationally excited electronic ground state of the 5'-GMP is produced and the longer-lived ( $c a .200 \mathrm{ps)}$ electronic excited state of $5^{\prime}-\mathrm{GMPD}^{+}$is also clearly resolved. The kinetic behaviour (Table 1) is that expected for the separate $5^{\prime}-\mathrm{GMP}$ and $5^{\prime}-\mathrm{GMPD}^{+}$.

Incorporation of 5'-GMP into a polymeric structure places the guanine base in a new environment in terms of hydrogen bonding, stacking interactions and solvation, which are all dependent on the biopolymer's secondary structure. Circular dichroism is particularly sensitive to secondary structure and has the advantage of an extensive library of diagnostic band positions. The buffered poly $(\mathrm{G})$ sample was found to have a negative band at $240 \mathrm{~nm}$ and a positive band at $260 \mathrm{~nm}$. These CD signals are characteristic of parallel G4-DNA sequences of the $G_{n}$ type. ${ }^{21}$ This secondary structure, in particular the formation of stacked guanine tetrads, results in major changes in the ground state IR and the ps-TRIR behaviour, with the dominating feature being a narrow carbonyl band at $1690 \mathrm{~cm}^{-1} \cdot{ }^{3,20}$ A biexponential decay is observed with a short-lived transient $(3.7 \pm 0.5 \mathrm{ps})$ and an additional longer-lived transient ( $36 \pm 4 \mathrm{ps)}$. This can be understood in terms of the new environment of the guanine base in the polymer, which is different to that of the monomer in a number of ways. Importantly, both the solvation and hydrogen bonding arrangements are altered. Furthermore, base-stacking may lead to electronic coupling due to orbital overlap. In the case of poly $(G)$ this manifests itself in a redshift in the UV absorption spectrum (see ESI $\dagger$ ) ${ }^{26}$ Longer-lived transient signals are commonly found for stacked nucleotides and have been ascribed to excimer-type excited states.

Moving from neutral to acidic solution is expected to result in a disruption of the Hoogsteen hydrogen bonding that is present in the G4-DNA. The CD spectra (Fig. 4) are consistent with a change in structure which has previously been attributed to the formation of a protonated double-stranded form. ${ }^{22}$ Therefore, not only is the chemical nature of the guanine bases expected to differ from that of $\operatorname{poly}(\mathrm{G})$, the structural environment will also be changed. The FTIR of poly $(\mathrm{G})$ in acid solution shows distinctly different features from those of the polynucleotide in neutral solution. The dominant bands resemble those of protonated 5'-GMP, with some 
evidence of bands similar to unprotonated 5'-GMP (e.g. bleach bands at $c a .1675 \mathrm{~cm}^{-1}$ and at $c a .1580 \mathrm{~cm}^{-1}$ and transients at $c a$. $1550 \mathrm{~cm}^{-1}$ and $c a .1640 \mathrm{~cm}^{-1}$ ). It is possible therefore that under these acid conditions some guanine residues are not protonated. Analysis of the recovery kinetics for the corresponding bleaches in the ps-TRIR reveal a fast and a slow phase of the ground state recovery. The longer recovery has the same rate constant (within error) as that observed for the transient band at $1587 \mathrm{~cm}^{-1}$ with decay times of $220 \pm 20$ ps for $5^{\prime}$-GMP in acid and $233 \pm 18$ ps for $\operatorname{poly}(\mathrm{G})$ in acid.

Finally one may note a major difference in the transient absorptions between the monomeric $5^{\prime}-\mathrm{GMPH}^{+}$and the protonated polymer. Thus while $5^{\prime}-\mathrm{GMPH}^{+}$shows two distinct transient absorption bands at 1520 and at $1640 \mathrm{~cm}^{-1}$, only the latter band is clearly defined in the protonated polymer. The reason for this is unclear and will require further study.

\section{Conclusions}

This study demonstrates the ability of ps-TRIR to unravel the complexities of medium effects on the excited states of nucleic acids, and in particular how protonation of guanine affects the photophysical properties of the compounds on both mononucleotide and polymeric systems where base-stacking and WatsonCrick base-pairing can be significant. The different dynamics observed between the non-protonated and protonated form of guanine nucleotide have long been recognised, because of the latter's strongly luminescent behaviour. This work reveals that the protonated excited state has characteristic vibrational absorption bands, which should assist computational chemists to determine the structure and electronic properties of this excited state. The results reported here for $5^{\prime}-\mathrm{H}_{2} \mathrm{GMP}$ in unbuffered solution demonstrate a particular strength of ps-TRIR by allowing for the resolution of both the ground state depletion and the transient behaviour of a mixture (namely the unprotonated and protonated forms).

It is well known that the photochemical behaviour of the polymeric forms of nucleotides is particularly complicated. Our study with poly $(\mathrm{G})$ reveals how the aggregation of the individual guanine moieties markedly changes the photophysical properties. The structure of poly $(\mathrm{G})$ is known to be complex with the formation of guanine tetrads being a major (but not the only) feature. We have analysed the transient kinetics with a biexponential function, although it is likely that this is an approximation. The short-lived species is ascribed to the vibrationally hot ground state, indicating that sub-picosecond processes (most probably internal conversion) play a major role. The longer-lived transient is assigned to an excimer-like state, the confirmation of which would require luminescence studies which were not possible in the current programme.

Finally, we have examined the case of protonated poly $(\mathrm{G})$ where both protonated and unprotonated guanines are expected to be present and the extent of stacking compared to that of $\operatorname{poly}(\mathrm{G})$ in neutral solution is much reduced. This additional complexity makes it difficult at this stage, without computational support, to explain all the spectral features we observe. However, it is possible to identify the modes that are most influenced by protonation and determine their dynamics, which must be directly related to structural modifications and changes in solvent coupling.

\section{Acknowledgements}

We are grateful to the EU access programme of the Rutherford Appleton Laboratory (JMK) which allowed these measurements to be made. We thank Dr Kate Ronayne for her help in making these measurements. We acknowledge financial support from Science Foundation Ireland (07/RFP/CHEF437).

\section{Notes and references}

1 M. K. Kuimova, J. Dyer, M. W. George, D. C. Grills, J. M. Kelly, P. Matousek, A. W. Parker, X. Z. Sun, M. Towrie and A. M. Whelan, Monitoring the effect of ultrafast deactivation of the electronic excited states of DNA bases and polynucleotides following $267 \mathrm{~nm}$ laser excitation using picosecond time-resolved infrared spectroscopy, Chem. Commun., 2005, 1182-1184.

2 S. Quinn, G. W. Doorley, G. W. Watson, A. J. Cowan, M. W. George, A. W. Parker, K. L. Ronayne, M. Towrie and J. M. Kelly, Ultrafast IR spectroscopy of the short-lived transients formed by UV excitation of cytosine derivatives, Chem. Commun., 2007, 2130-2132.

3 D. A. McGovern, S. Quinn, G. W. Doorley, A. M. Whelan, K. L. Ronayne, M. Towrie, A. W. Parker and J. M. Kelly, Picosecond infrared probing of the vibrational spectra of transients formed upon UV excitation of stacked G-tetrad structures, Chem. Commun., 2007, 51585160 .

4 M. K. Kuimova, A. J. Cowan, P. Matousek, A. W. Parker, X. Z. Sun, M. Towrie and M. W. George, Monitoring the direct and indirect damage of DNA bases and polynucleotides by using time-resolved infrared spectroscopy, Proc. Nat. Acad. Sci. U. S. A., 2006, 103, 2150 2153.

5 B. Elias, C. Creely, G. W. Doorley, M. M. Feeney, C. Moucheron, A. Kirsch-DeMesmaeker, J. Dyer, D. C. Grills, M. W. George, P. Matousek, A. W. Parker, M. Towrie and J. M. Kelly, Photooxidation of guanine by a ruthenium dipyridophenazine complex intercalated in a doublestranded polynucleotide monitored directly by picosecond visible and infrared transient absorption spectroscopy, Chem.-Eur. J., 2008, 14, 369.

6 W. J. Schreier, T. E. Schrader, F. O. Koller, P. Gilch, C. E. CrespoHernández, V. N. Swaminathan, T. Carell, W. Zinth and B. Kohler, Thymine dimerization in DNA is an ultrafast photoreaction, Science, 2007, 315, 625-629.

7 M. Hare, C. T. Middleton, K. I. Mertel, J. M. Herbert and B. Kohler, Time-resolved infrared spectroscopy of the lowest triplet state of thymine and thymidine, Chem. Phys., 2008, 347, 383-392.

8 G. W. Doorley, D. A. McGovern, M. W. George, M. Towrie, A. W. Parker, J. M. Kelly and S. J. Quinn, Picosecond transient infrared study of the ultrafast deactivation processes of electronically excited B-DNA and Z-DNA forms of [poly(dG-dC) $]_{2}$, Angew. Chem., Int. Ed., 2009, 48, 123-128.

9 M. Towrie, D. C. Grills, J. Dyer, J. A. Weinstein, P. Matousek, R. Barton, P. D. Bailey, N. Subramaniam, W. M. Kwok, C. Ma, D. Phillips, A. W. Parker and M. W. George, Development of a broadband picosecond infrared spectrometer and its incorporation into an existing ultrafast time-resolved resonance Raman, UV/visible, and fluorescence spectroscopic apparatus, Appl. Spectrosc., 2003, 57, 367-380.

10 D. Onidas, D. Markovitsi, S. Marguet, A. Sharonov and T. Gustavsson, Fluorescence Properties of DNA Nucleosides and Nucleotides: A Refined Steady-State and Femtosecond Investigation, J. Phys. Chem. $B, 2002,106,11367-11374$.

11 W.-M. Kwok, C. Ma and D. L. Phillips, Femtosecond time- and wavelength- resolved fluorescence and absorption spectroscopic study of the excited states of adenosine and an adenine oligomer, $\mathrm{J} . \mathrm{Am}$. Chem. Soc., 2006, 128, 11894-11905.

12 C. E. Crespo-Hernández, B. Cohen, P. M. Hare and B. Kohler, Ultrafast excited-state dynamics in nucleic acids, Chem. Rev., 2004, 104, 19772020.

13 C. E. Crespo-Hernandez, B. Cohen and B. Kohler, Base stacking controls excited state dynamics in A-T DNA, Nature, 2005, 436, 114144.

14 P. O’Neill, A. W. Parker, M. A. Plumb and L. D. A. Siebbeles, Guanine modifications following ionization of DNA occurs predominantly via intra- and not interstrand charge migration: an experimental and theoretical study, J. Phys. Chem. B, 2001, 105, 5283-5290. 
15 J. Peon and A. H. Zewail, DNA/RNA nucleotides and nucleosides: direct measurement of excited-state lifetimes by femtosecond fluorescence up-conversion, Chem. Phys. Lett., 2001, 348, 255-262.

16 J. M. L. Pecourt, J. Peon and B. Kohler, DNA Excited-state dynamics: ultrafast internal conversion and vibrational cooling in a series of nucleosides, J. Am. Chem. Soc., 2001, 123, 10370-10378.

17 B. Cohen, P. M. Hare and B. Kohler, Ultrafast excited state dynamics of adenine and monomethylated adenines in solution: implications for the nonradiative decay mechanism, J. Am. Chem. Soc., 2003, 125, 13594 13601.

18 T. Fujiwara, Y. Kamoshida, R. Morita and M. Yamashita, Temporallyseparated ultrafast-emission dynamics of neutral and cationic molecules in guanine and its derivative aqueous solutions, J. Photochem. Photobiol., B, 1997, 41, 114-121.

19 J. T. Davis, G-Quartets 40 years later: from 5'-GMP to molecular biology and supramolecular chemistry, Angew. Chem., Int. Ed., 2004, 43, 668-698.

20 (a) M. R. Guzman, J. Liquier, S. K. Brahmachari and E. Taillandier, Characterization of parallel and antiparallel G-tetraplex structures by vibrational spectroscopy, Spectrochim. Acta, Part A, 2006, 64A, 495-503; (b) R. Ghana, C. Walss and J. A. Walmsley, Sodium and potassium ion-promoted formation of supramolecular aggregates of $2^{\prime}$-deoxyguanylyl-(3'-5')-2'-deoxyguanosine, J. Biomol. Struct. Dyn., 1996, 14, 101-110.

21 (a) G. Gottarelli, S. Lena, S. Masiero, S. Pieraccini and G. P. Spada, The Use of Circular Dichroism Spectroscopy for Studying the Chiral
Molecular Self-Assembly: An Overview, Chirality, 2008, 20, 471-485; (b) R. Giraldo, M. Suzuki, L. Chapman and D. Rhodes, Promotion of parallel DNA quadruplexes by a yeast telomere binding protein: A circular dichroism study, Proc. Natl. Acad. Sci. U. S. A., 1994, 91, 7658-7662; (c) D. Gray, A circular dichroism study of poly dG, poly dC, and poly dG:dC, Biopolymers, 1974, 13, 2087-2102.

22 E. A. Lesnik, I. M. Kochkina, A. S. Tikhonenko and Y. M. Varshavskii, Structure of polyriboguanylic acid in solution, Mol. Biol. (Moscow), 1980, 14, 820-829.

23 (a) D. Markovitsi, F. Talbot, T. Gustavsson, D. Onidas, E. Lazzarotto and S. Marguet, Molecular spectroscopy:Complexity of excited-state dynamics in DNA, Nature, 2006, 441, E7; (b) D Markovitsi, T. Gustavsson and F. Talbot, Perspective: Excited states and energy transfer among DNA bases in double helices, Photochem. Photobiol. Sci., 2007, 6, 717.

24 G. Barbarella, M. L. Capobianco, A. Carcuro, F. P. Colonna, A. Garbesi and V. Tignoli, ${ }^{15} \mathrm{~N}-{ }^{13} \mathrm{C}$ Nuclear Magnetic Resonance of Deoxydinucleotide Monophosphates. I. Protonation, of Deoxycytidylyl$\left(3^{\prime}, 5^{\prime}\right)$-guanosine in dimethylsuphoxide, Can. J. Chem., 1988, 66, $2492-$ 2497.

25 C. T. Middleton, B. Cohen and B. Kohler, Solvent and solvent isotope effects on the vibrational cooling dynamics of a DNA base derivative, J. Phys. Chem. A, 2007, 111, 10460-10467.

26 D. Markovitsi, T. Gustavsson and A. Sharonov, Cooperative effects in the photophysical properties of self-associated triguanosine diphosphates, Photochem. Photobiol., 2004, 79, 526-530. 